\title{
A conjecture on the existence of common quadratic Lyapunov functions for positive linear systems
}

\author{
Oliver Mason ${ }^{1} \quad$ Robert Shorten ${ }^{2}$
}

\begin{abstract}
We present a conjecture concerning necessary and sufficient conditions for the existence of a common quadratic Lyapunov function (CQLF) for a switched linear system obtained by switching between two positive linear time-invariant (LTI) systems. We conjecture that these conditions are also necessary and sufficient for the exponential stability of such switched linear systems; namely, the existence of a CQLF is a nonconservative stability condition in this case. A number of new results supporting this conjecture are described.
\end{abstract}

\section{Introduction}

The problem of determining necessary and sufficient conditions for the existence of a common quadratic Lyapunov function (CQLF) for a set of stable linear time-invariant (LTI) systems

$$
\Sigma_{A_{i}}: \dot{x}(t)=A_{i} x(t), A_{i} \in \mathbb{R}^{n \times n}, 1 \leq i \leq k
$$

plays an important role in the study of switched linear systems of the form:

$$
\dot{x}(t)=A(t) x(t), A(t) \in\left\{A_{1}, \ldots, A_{k}\right\} .
$$

Formally, if there is a symmetric positive definite matrix $P$ that simultaneously satisfies the Lyapunov inequalities

$$
A_{i}^{T} P+P A_{i}=-Q_{i}<0, i \in\{1,2, \ldots k\}
$$

then $V(x)=x^{T} P x$ is a CQLF for the system (I) and the associated LTI systems $\Sigma_{A_{i}}$. The existence of a CQLF is sufficient to guarantee global uniform exponential stability of (1) for arbitrary switching sequences. It is well known that requiring the existence of a CQLF for a switched linear system is, in general, a conservative stability condition [1]. However, it has recently been established that entire system classes exist for which the existence of a CQLF is not necessarily a conservative stability condition $[2,3]$. In view of this

\footnotetext{
${ }^{1}$ Hamilton Institute, NUI Maynooth, Ireland. Email:oliver.mason@may.ie
${ }^{2}$ Hamilton Institute, NUI Maynooth, Ireland. Email:robert.shorten@may.ie
}

0-7803-7896-2/03/\$17.00 @2003 IEEE observation, a problem of considerable interest and importance is to identify precisely those system classes for which the existence of a CQLF is a non-conservative stability condition. The work of this paper is primarily motivated by such considerations.

\section{Notation and Preliminaries}

For a matrix $A$ in $\mathbb{R}^{n \times n}, a_{i j}$ denotes the element in the $(i, j)$ position of $A$, and we shall write $A \succeq 0$ if $a_{i j} \geq 0$ for $1 \leq i, j \leq n$. The matrix $A \in \mathbb{R}^{n \times n}$ is said to be Hurwitz if all the eigenvalues of $A$ have negative real parts, and for $P$ in $\mathbb{R}^{n \times n}$ the notation $P>0$ means that the matrix $P$ is positive definite.

A matrix $A$ in $\mathbb{R}^{n \times n}$ is a Metzler matrix if all of the offdiagonal elements of $A$ are non-negative; that is $a_{i j} \geq 0$ for $i \neq j$. The LTI system $\Sigma_{A}$ is positive ${ }^{1}$ [4] if and only if $A$ is a Metzler matrix. The associated class of $M$-matrices $[5,6]$ is defined to consist of matrices $A$ with non-positive off-diagonal elements, all of whose eigenvalues lie in the open right half-plane.

\section{A conjecture:}

Let $\Sigma_{A_{i}}, i=1,2$ be a pair of stable positive LTI systems. Recent work carried out by the authors suggests that the matrix product $A_{1} A_{2}^{-1}$ having no negative eigenvalues is a necessary and sufficient condition for:

(i) the existence of a CQLF for the LTI systems $\Sigma_{A_{1}}$ $\Sigma_{A_{2}}$

(ii) global exponential stability of the switched linear system (1).

\section{Sufficient conditions for CQLF existence}

In this section we state without proof a number of sufficient conditions for a pairs of stable positive LTI systems to possess a CQLF. Details of the proofs can be found in [7]. The result stated in the next lemma is not new [8] but is included here for the sake of comparison with the main result of this note (Theorem 3.1 below).

\footnotetext{
${ }^{1}$ An LTI system is positive if, for any initial conditions where the state variables are all non-negative, the state variables remain non-negative for all time

Proceedings of the American Control Conference Denver, Colorado June 4-6, 2003
} 
Lemma 3.1 Let $\Sigma_{A_{1}}, \Sigma_{A_{2}}$ be stable positive LTI systems, with $A_{1}-A_{2} \succeq 0$. Then $\Sigma_{A_{1}}$ and $\Sigma_{A_{2}}$ have a CQLF $V(x)=x^{T} P x$, with $P$ diagonal.

Theorem 3.1 Let $\Sigma_{A_{1}}, \Sigma_{A_{2}}$ be stable positive LTI systems. If both $A_{1} A_{2}^{-1}$ and $A_{2}^{-1} A_{1}$ are M-matrices, then $\Sigma_{A_{1}}$ and $\Sigma_{A_{2}}$. have a CQLF, $V(x)=x^{T} P x$, and moreover, $P$ may be taken to be a diagonal matrix.

Note that within the class of matrices with non-positive off-diagonal elements, a non-singular matrix having no eigenvalues on the negative real axis is equivalent to it being an M-matrix ([5]).

Theorem 3.2 Let $\Sigma_{A_{1}}, \Sigma_{A_{2}}$ be stable positive LTI systems. Suppose that $A_{1} A_{2}^{-1} \succeq 0$ and $A_{2}^{-1} A_{1} \succeq 0$. Then $\Sigma_{A_{1}}$ and $\Sigma_{A_{2}}$ have a CQLF.

It was noted in [5] that if $A_{1}, A_{2}$ are both Hurwitz Metzler matrices with $A_{1} \succeq A_{2}$, then $A_{1} A_{2}^{-1}$ and $A_{2}^{-1} A_{1}$ are both M-matrices. Thus the class of matrices covered by Lemma 3.1 is a subclass of the class covered by Theorem 3.1. (In fact, Theorem 3.2 covers a still larger class of systems than Theorem 3.1.) The next example shows that it is a strict subclass.

Example: Consider the two Metzler matrices in $\mathbb{R}^{3 \times 3}$ given by

$$
\begin{aligned}
& A_{1}=\left(\begin{array}{ccc}
-1.1686 & 0.5618 & 0.3837 \\
0.9512 & -1.7425 & 0.7293 \\
0.9460 & 0.4830 & -1.8474
\end{array}\right) \\
& A_{2}=\left(\begin{array}{ccc}
-1.7697 & 0.3163 & 0.1496 \\
0.1599 & -0.9759 & 0.2794 \\
0.2167 & 0.1769 & -1.0543
\end{array}\right) .
\end{aligned}
$$

It is evident that neither $A_{1}-A_{2} \succeq 0$ nor $A_{2}-A_{1} \succeq 0$ is true, so Lemma 3.1 does not apply. However, it is a simple matter to check that both $A_{1} A_{2}^{-1}$ and $A_{2}^{-1} A_{1}$ are M-matrices. Thus by Theorem 3.1 we can conclude that $A_{1}$ and $A_{2}$ have a CQLF $x^{T} P x$ with $P$ diagonal.

In [9] it is shown that LTI systems whose system matrices commute have a CQLF $x^{T} P x$. The next result shows that $P$ may be chosen to be diagonal if the LTI systems are positive.

Theorem 3.2 Let $\Sigma_{A_{1}}, \Sigma_{A_{2}}$ be two positive LTI systems with $A_{1} A_{2}=A_{2} A_{1}$. Then there is a CQLF $V(x)=x^{T} P x$ for $\Sigma_{A_{1}}, \Sigma_{A_{2}}$ with $P$ diagonal.

\section{Conclusions}

In this paper, we have proposed a conjecture concerning CQLF existence for a pair of stable positive LTI systems. It was also conjectured that for switched linear systems obtained by switching between stable positive ITI systems, the existence of a CQLF is a nonconservative stability criterion. A number of new results in this direction were presented. The authors have also gathered considerable empirical evidence supporting the conjecture.

Acknowledgements: This work was partially supported by the European Union funded ' research training network Multi-Agent Control, HPRN-CT1999-00107 $7^{2}$ and by the Enterprise Ireland grant SC/2000/084/Y. Neither the European Union or Enterprise Ireland is responsible for any use of data appearing in this publication.

\section{References}

11] W. P. Dayawansa and C. F. Martin, "A converse Lyapunov theorem for a class of dynamical systems which undergo switching," IEEE Transactions on Automatic Control, vol. 44, pp. 751-760, 1999.

[2] R. N. Shorten and K. Narendra, "Necessary and sufficient conditions for the existence of a common quadratic Lyapunov function for a finite number of stable second order linear time-invariant systems," International Journal of Adaptive Control and Signal Processing, In Press.

[3] R. Shorten, F. Ó Cairbre, and P. Curran, "On the dynamic instability of a class of switching systems," in Proceedings of IFAC conference on Artificial Intelligence in Real Time Control, 2000.

[4] L. Farina and S. Rinaldi, Positive linear systems. Wiley Interscience Series, 2000.

[5] R. Horn and C. Johnson, Topics in matrix analysis. Cambridge University Press, 1991.

[6] D. Stipanovic and D. Siljak, "Stability of polytopic systems via convex M-matrices and parameterdependent Lyapunov functions," Nonlinear Analysis, vol. 40, pp. 589-609, 2000.

[7] O. Mason and R. Shorten, "A conjecture on the existence of common quadratic Lyapunov functions for positive linear systems," tech. rep., NUIM SS/2002/09, 2002 .

[8] Y. Mori, T. Mori, and Y. Kuroe, "On a class of linear constant systems which have a common quadratic Lyapunov function," in Proceedings of 37 th Conference on Decision and Control, 1998.

[9] K. Narendra and J. Balakrishnan, "A Common Lyapunov Function for Stable LTI Systems with Commuting A-Matrices," IEEE Transactions on Automatic Control, vol. 39, no. 12 , pp. 2469-2471, 1994.

\footnotetext{
${ }^{2}$ This work is the sole responsibility of the authors and does not reflect the European Union's opinion
} 